\title{
Vibration based diagnosis of wheel defects of metro train sets using one period analysis on the wayside
}

\author{
Onur Kılınç ${ }^{1}$, Jakub Vágner ${ }^{2}$ \\ Department of Transport Means and Diagnostics, Faculty of Transport Engineering, \\ University of Pardubice, Pardubice, Czech Republic \\ ${ }^{1}$ Corresponding author \\ E-mail: ${ }^{1}$ onur_kilinc@anadolu.edu.tr, ${ }^{2}$ jakub.vagner@upce.cz \\ Received 14 April 2017; accepted 15 April 2017 \\ DOI https://doi.org/10.21595/vp.2017.18492
}

Check for updates

\begin{abstract}
This research examines two different methods; Wavelet Packet Energy (WPE) and Time-domain Features (TDF) which are effective in faulty signal feature extraction of metro wheels in wayside level using vibration sensors. Signals of each wheelset passing of a trainset with both healthy and faulty wheels are recorded by the vibration sensors which are mounted on both left and right rails and a novel one-period sampling is performed at $51.2 \mathrm{kHz}$ sample rate. Retrieved signal samples are used in the construction of a database which is consistent of healthy and faulty cases. Since the database has insufficient number of faulty samples, the database is balanced by a method so called Adaptive Synthetic Sampling (ADASYN) so that each class has the same number of observations. Two state-of art classifiers; Support Vector Machines (SVM) and Fisher Linear Discriminant Analysis (FLDA) are employed by utilizing 16-fold cross validation to solve the two-class problem. Referring to the results, SVM-I-TDF outperforms by classifying all samples with a success rate of $100 \%$ and other methods have also promising results. Proposed methods may be used in the condition monitoring of metro wheelsets effectively by means of not only performance but also cost-efficiency.
\end{abstract}

Keywords: wavelet packet energy, time domain features, wheelset fault diagnosis, one period analysis, Fisher linear discriminant analysis, support vector machine.

\section{Introduction}

Condition monitoring of railway vehicles is advantageous due to providing cost efficient maintenance especially when a wayside diagnosis approach is used. The main challenge is to make the diagnosis of the related component without significant number of false positives and false negatives; otherwise irreversible and high costs may occur.

Diagnosis of faults of metro wheelsets is vital to prevent the damage on other structures of the vehicle and rails which may threaten the safety of the run. Detection of these faults provides cost effective maintenance outperforming the scheduled maintenance by means of cost efficiency. The most successful detection of such cases can be performed by mounting sensors of each wheelsets which is too costly. For that reason, wayside approaches have been focused by the researches in recent years.

In condition monitoring of railway vehicles, three main categories are existed; model based dynamical techniques including various Kalman [1,2] and particle filter [3], signal based techniques including band-pass filter, spectral analysis, wavelet analysis and Fast Fourier Transform [4], and wayside sensor based approaches which are generally practical in wheel defects according to a recent review [5]; using accelerometer and piezoelectric sensors on rail and using wavelet based methods and thresholding to identify the degree of the wheel flat [6], using a high speed camera to identify the wheel profile when the railway vehicle is passing by a low speed (10 $\mathrm{mph}$ ) and image analysis, using optical sensors, accelerometers, load cells and strain gages to measure vertical deflection of the rail which makes it able to identify wheel defects like; out-of round, flat, shelling, measuring lateral force to determine bogie performance using hunting track detectors [7], acoustic bearing defect detectors which is based on statistical processing of the data, ultrasonic cracked wheel detection and many others [8]. 
The main drawback of wayside diagnosis approaches is noise since acting of multiple components produce irrelevant vibration and environmental noise which is relatively heavy in most cases. Moreover, when it comes to vibration analysis of metro wheelsets, the difficulty of distinguishing faulty data can be more challenging since the signals are badly affected by both noise and insufficiency of the sampling time due to the fact that the wheelset which is wanted to be diagnosed is overlapping to other sampling windows of nearby wheels of the same bogie.

This study presents several methods which are efficient in wheel defect detection of metro train sets. Throughout the research, measurements from two vibration sensors which are accompanied by two optical gates to detect vehicle speed and wheelset positions are recorded on a passage between two metro stations which are present in Prague metro line. Two feature extraction techniques are proposed which are considered as efficient in non-stationary diagnosis besides two cutting edge classifiers to maintain a stable condition monitoring framework.

The organization of this paper is as follows: Test environment and signal acquisition as well as sampling by one period analysis are told in Section 2. Feature extraction procedures for each proposed method is presented in Section 3. Experimental results and discussion is given in Section 4. Lastly, conclusion part is presented in Section 5.

\section{Database and segmentation}

In this research, a vibration based measurement system is installed in a metro tunnel on a wayside passage between Dejvická and Bořislavka which is located in Prague metro line-A. Two vibration sensors $Z_{1}$ and $Z_{2}$ are accompanied by two optical gates $G_{A}$ and $G_{B}$ operating at $500 \mathrm{~Hz}$ for determining wheelset positions and vehicle speed.

The wayside passage, which all measurements are done, provides a stable speed of the run which is almost constant. Thanks to the instrumentation device NI-cDAQ-9234 which records the signals in all channels at $51.2 \mathrm{kHz}$ sampling rate during all day when metros are in operation. Measurements are done in the project; Competence Centre of Railway Vehicles, No. TAČR TE01020038.

Metro train sets of type $81-71 \mathrm{M}$ are identical by means of bogies and passenger cars (4-axle, five passenger cars). During one day, eight passing of a train set (ID-108) which both have wheel defects and normal wheelsets. The wayside measurement system is shown in Fig. 1. The sampling window is chosen so that each wheel is rotated only one period according to the wheel diameter information from maintenance of Prague metro and exact operational speed.

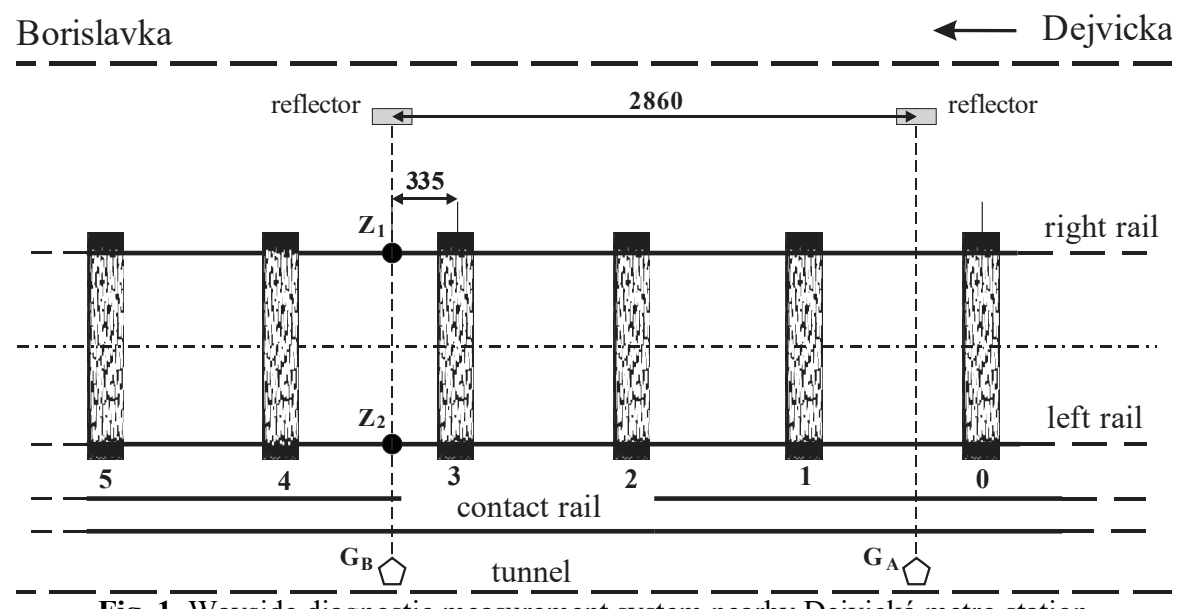

Fig. 1. Wayside diagnostic measurement system nearby Dejvická metro station

Two main datasets are prepared in order to evaluate efficiency of proposed methods; A1 has only measurement signals of normal and faulty cases of wheels while SA1 is consistent of both 
measured faulty samples and over sampled vectors of faulty wheels. Table 1 describes the datasets.

Table 1. Description of classified datasets of measured and synthesized datasets

\begin{tabular}{|c|c|c|c|c|c|c|}
\hline $\begin{array}{c}\text { Train } \\
\text { ID. }\end{array}$ & Condition & $\begin{array}{c}\text { Measured } \\
\text { samples }\end{array}$ & $\begin{array}{c}\text { Synth. } \\
\text { samples }\end{array}$ & $\begin{array}{c}\text { Total normal } \\
\text { samples }\end{array}$ & $\begin{array}{c}\text { Wheelset } \\
\text { interval }\end{array}$ & Label \\
\hline \multirow{4}{*}{108} & Normal & 16 & 0 & \multirow{2}{*}{32} & $1-4,11-14$ & \multirow{2}{*}{ A1 } \\
\hline & Faulty & 16 & 0 & & 7 & \\
\hline & Normal & 128 & 0 & \multirow{2}{*}{256} & $1-4,11-14$ & \multirow{2}{*}{ SA1 } \\
\hline & Faulty & 16 & 112 & & 7 & \\
\hline
\end{tabular}

\section{Proposed approaches}

In this section, the information about proposed techniques for extracting faulty characteristics of the measured signals, which are retrieved in wayside passage by vibration sensors, are told. Furthermore, an adaptive oversampling algorithm for more reliable model evaluation is also presented.

\subsection{Wavelet packet energy}

This method is based on Short Time Fourier Transform (STFT) which is used in frequency domain signal processing applications.

In Wavelet Packet Transform (WPT) Fourier spectrum of the signal is divided into desired number of frequency bands which provides custom frequency resolution. Respect to the number of level $L$, number of components in WPT is $n=2^{L}$. After determining maximum number of level, filtering for each frequency band is carried out on the signal to obtain low and high frequency components which is followed by down sampling to achieve next level. Referring to Fig. 2, since WPT is lack of being time-invariant [11], energy property of each wavelet packet is calculated to increase its performance in detecting transients in non-stationary signal processing which leads to Wavelet Packet Energy (WPE) feature extraction (Fig. 3).

In this research, Haar wavelets [12] is preferred in the decomposition of using WPE. Five level WPE is calculated which results size of $32 \times 1$ feature vectors for each signal sample.
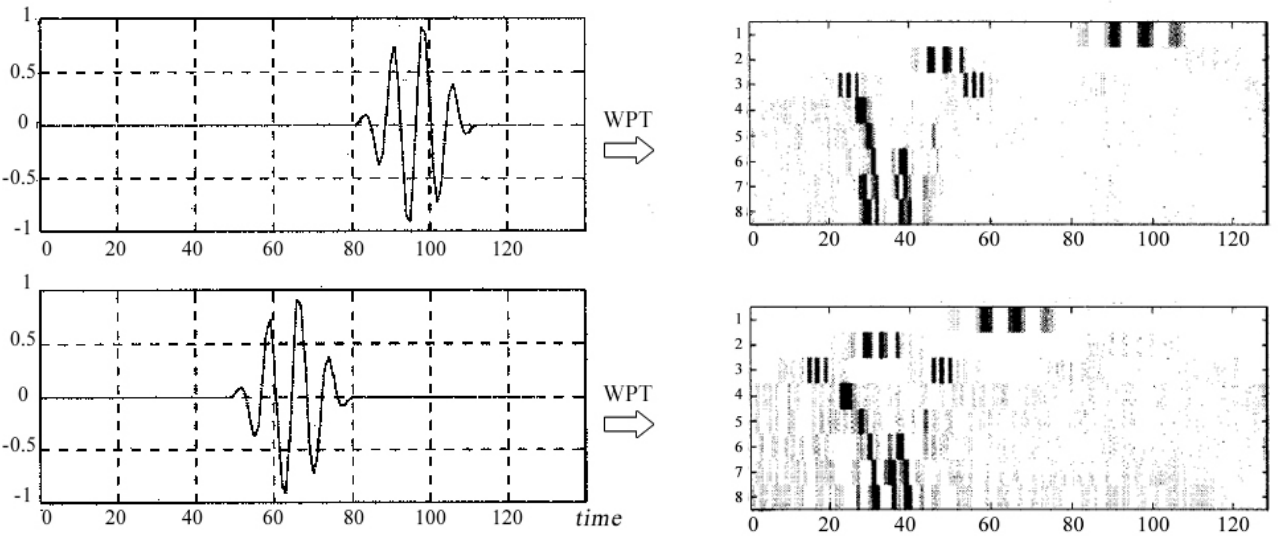

a)

b)

Fig. 2. a) Transient signals that are shifted in time domain, b) resultant three level WPT

\subsection{Time domain features}

Statistically, faulty signals have similar behaviour not only in frequency domain but also in time domain. Statistical time-domain feature extraction methods have already been proposed for 
recognition of numerous faulty conditions of different components in rotating machinery [13, 14$]$.

Proposed Time Domain Features (TDF) are consistent of several individual properties (Eq. (1)) of the discrete signals which is preferred as in the research [10]:

$T D F=[\text { energy, mean, std. dev, } \max , \min , \text { kurtosis, skewness, crest factor }]_{8 \times 1}$.

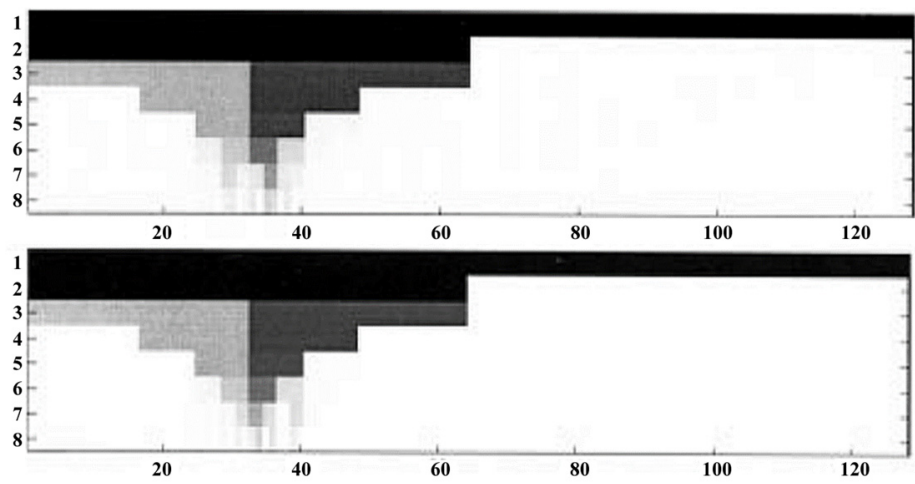

Fig. 3. Resultant three level WPE for the signals in Fig. 2

\subsection{Adaptive synthetic sampling}

In practical applications of wayside fault diagnosis subjected to railway vehicles, number of healthy cases superimpose the faulty ones in the measurement. However, most classifiers in pattern recognition requires balanced database which means the same number of observations in each class. When the faulty observations are insufficient, which means the number of features in each vector is shorter than the number of faulty samples, model evaluation cannot be performed in a stable way. The most straightforward way to deal with this problem is to interpolate samples and generate new ones.

According to recent literature, a synthetic minority oversampling technique (SMOTE) is proposed [15] which interpolates the existing feature vectors of the minority class linearly and create new feature vectors. Nevertheless, SMOTE approach has limited to focusing only on minority class. Being lack of consideration of the boundaries between classes, over sampling with SMOTE has unrealistic results.

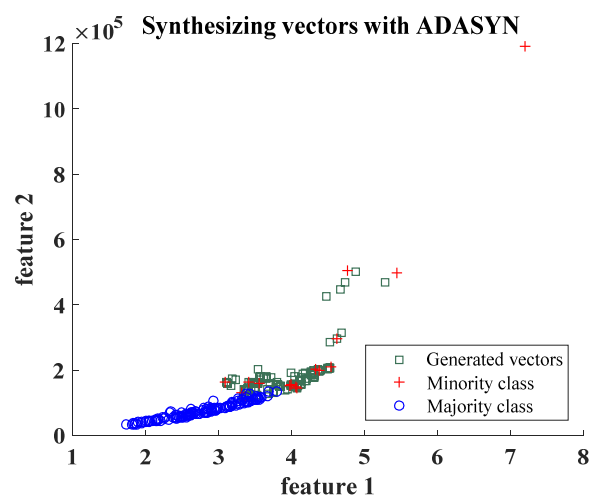

Fig. 4. Feature oversampling from minority class to balance datasets using ADASYN (only two dimensions are shown)

In the proposed method for generating additional feature vectors, Adaptive Synthetic Sampling (ADASYN) [16], which is the further improvement of SMOTE is used. ADASYN methodology 
creates more samples between boundaries of each class adaptively for each feature, thus provides more stable oversampling.

The oversampling operation of time domain features which are extracted from samples of wheel defects from 16 observations (minority class) to the number of normal observations (majority class that has 128 observations) is shown in Fig. 4. It is clear that, the outlier effect is diminished and samples are created in a more plausible way.

\section{Experimental results}

After having extracted the features with proposed WPE and TDF algorithms, two state of art classifiers; Support Vector Machine with linear kernel (SVM-I) and Fisher Linear Discriminant Analysis (FLDA) are employed in the analyses of measured and synthetically oversampled datasets of normal and faulty cases using different number of cross validations that leads to best results. All classification results are shown in Table 2.

Referring to the results, in case of insufficient data, WPE approach is nominated to be not very promising whereas after oversampling the classification accuracy increased significantly. Best results are obtained by TDF with SVM-I classifier in both insufficient and over sampled datasets.

Table 2. Classification performance of measured (A1) and synthesized classes (SA1)

\begin{tabular}{|c|c|c|c|c|c|}
\hline \multirow{2}{*}{ Classifier } & \multicolumn{5}{|c|}{ Average classification accuracies (\%) } \\
\cline { 2 - 4 } & WPE & TDF & Num. of cross validation & Sensors & Labels \\
\hline \multirow{2}{*}{ FLDA } & 68.6 & 100 & 8 & & A1 \\
\cline { 2 - 4 } \cline { 6 - 6 } & 95.7 & 99.2 & 16 & Z1-Z2 & SA1 \\
\cline { 2 - 4 } SVM-I & 90.6 & 100 & 8 & A1 \\
\cline { 2 - 4 } & 99.6 & 100 & 16 & SA1 \\
\hline
\end{tabular}

\section{Conclusions}

In this study, two techniques that are efficient in non-stationary signal processing are used in order to diagnose wheel defects on metro train sets.

Measurement of real faulty data is so rare in real operation of metro trainsets unless a real-time measurement is performed. After having utilized an adaptive over sampling method (ADSAYN), more faulty samples are acquired which make it able to understand if more normal samples can be classified correctly.

With respect to results, an efficient model is developed after over sampling data and all normal cases are managed to be classified by both methods efficiently up to $100 \%$ (SVM-I-TDF). This research may aid the maintenance specialists by providing an efficient framework for wayside condition monitoring of railway vehicles.

\section{References}

[1] Tipireddy R., Nasrellah H. A., Manohar C. S. A Kalman filter based strategy for linear structural system identification based on multiple static and dynamic test data. Probabilistic Engineering Mechanics, Vol. 24, Issue 1, 2009, p. 60-74.

[2] Hide C., Moore T., Smith M. Adaptive Kalman filtering algorithms for integrating GPS and low cost INS. Position Location and Navigation Symposium, 2004, p. 227-233.

[3] Charles G., Goodall R., Dixon R. Model-based condition monitoring at the wheel-rail interface. Vehicle System Dynamics, Vol. 46, Issue 1, 2008, p. 415-430.

[4] Bruni S., Goodall R., Mei T. X., Tsunashima H. Control and monitoring for railway vehicle dynamics. Vehicle System Dynamics, Vol. 45, Issues 7-8, 2007, p. 743-779.

[5] Ngigi R. W., Pislaru C., Ball A., Gu F. Modern techniques for condition monitoring of railway vehicle dynamics. Journal of Physics: Conference Series, Vol. 364, 2012, p. 012016.

[6] Belotti V., Crenna F., Michelini R. C., Rossi G. B. Wheel-flat diagnostic tool via wavelet transform. Mechanical Systems and Signal Processing, Vol. 20, Issue 8, 1953, p. 1966-2006. 
[7] Barke D., Chiu W. K. Structural health monitoring in the railway industry: a review. Structural Health Monitoring, Vol. 4, Issue 1, 2005, p. 81-93.

[8] Brickle B., Morgan R., Smith E., Brosseau J., Pinney C. Identification of Existing and New Technologies for Wheelset Condition Monitoring. RSSB Report T607 UK P-07-005, TTCI Ltd., 2008.

[9] Lou X., Loparo K. A. Bearing fault diagnosis based on wavelet transform and fuzzy inference. Mechanical Systems and Signal Processing, Vol. 18, Issue 5, 2004, p. 1077-1095.

[10] Yu X., Ding E., Chen C., Liu X., Li L. A novel characteristic frequency bands extraction method for automatic bearing fault diagnosis based on Hilbert-Huang transform. Sensors, Vol. 15, Issue 11, 2015, p. 27869-27893.

[11] Yen G. G., Lin K.-C. Wavelet packet feature extraction for vibration monitoring. IEEE Transactions on Industrial Electronics, Vol. 47, Issue 3, 2000, p. 650-667.

[12] Li L., Qu L., Liao X. Haar wavelet for machine fault diagnosis. Mechanical Systems and Signal Processing, Vol. 21, Issue 4, 2007, p. 1773-1786.

[13] Fu S., Liu K., Xu Y., Liu Y. Rolling bearing diagnosing method based on time domain analysis and adaptive fuzzy C-means clustering. Shock and Vibration, Vol. 1, 2016, p. 2016-8.

[14] Wang M., Hu N.-Q., Hu L., Gao M. Feature optimization for bearing fault diagnosis. International Conference on Quality, Reliability, Risk, Maintenance, and Safety Engineering, 2013, p. 1738-1741.

[15] Chawla N. V., Bowyer K. W., Hall L. O., Kegelmeyer W. P. SMOTE: synthetic minority over-sampling technique. Journal of Artificial Intelligence Research, Vol. 16, 2002, p. 321-357.

[16] He H., Bai Y., Garcia E. A., Li S. ADASYN: Adaptive synthetic sampling approach for imbalanced learning. IEEE International Joint Conference on Neural Networks, IEEE World Congress on Computational Intelligence, 2008, p. 1322-1328. 